\title{
ADAPTIVE TARGET DETECTION ACROSS A CLUTTER BOUNDARY: GLR AND MAXIMALLY INVARIANT DETECTORS
}

\author{
Hyung Soo Kim and Alfred O. Hero \\ Department of Electrical Engineering and Computer Science \\ University of Michigan, Ann Arbor, MI 48109-2122
}

\begin{abstract}
We present and compare adaptive detection algorithms developed for synthetic aperture radar (SAR) targets in structured clutter, utilizing both generalized likelihood ratio (GLR) tests and maximal invariant (MI) tests. We consider the problem of detecting a target straddling a known boundary between two independent clutter regions inducing a clutter covariance matrix with block diagonal structure. GLR and MI tests are presented for various clutter scenarios: two totally unknown clutter types, one of the clutter types known except for its variance, and one of the clutter types completely known. Numerical comparisons will illustrate that GLR tests and MI tests are complementary-neither test strategy uniformly outperforms the other-suggesting that it may be worthwhile to hybridize these tests for overall optimal performance.
\end{abstract}

\section{INTRODUCTION}

In automatic target recognition, the most important issue is reliable detection which is robust to target and clutter variability, yet maintains the highest possible discrimination capability. In the past, most adaptive radar and array detection problems have been formulated under the general assumption that the target has known form but unknown amplitude in Gaussian noise whose covariance matrix is totally unknown or unstructured. The nature of this assumption led to the application of the generalized multivariate analysis of variance (GMANOVA) model to the measurements, and the subsequent development of many detection algorithms. With this assumption and the GMANOVA model, Kelly [1] derived the constant false alarm rate (CFAR) test using the generalized likelihood ratio (GLR) principle and in so doing proved that the test is an optimal maximal invariant (MI) test as well.

However, when a prior structure of the clutter covariance matrix exists, one can expect an improvement in performance by exploiting this a priori structure. Also when

This work was supported in part by AFOSR under MURI grant: F49620-97-0028. tractable, the reduced parameterization of the structured covariance can be introduced and the GLR test can be applied. For adaptive arrays, Bose and Steinhardt [2] proposed an MI detector which outperforms the Kelly's test when the clutter covariance matrix is assumed to have a certain known block diagonal structure. This work was the springboard in [3] for synthetic aperture radar (SAR) imaging target detection in the difficult deep hide scenario where the target parks along a known boundary separating two adjacent clutter regions. Indeed, under the assumption that the two clutter types are statistically independent, the spatial covariance has a similar block diagonal structure to that in [2] and this structure was used in [3] to derive another MI test.

In this paper we extend the work reported in [3] by deriving the GLR test and comparing the invariant methods of [2] and [3]. Derivations of the GLR and MI test statistics for the case of structured covariance are not trivial, and the details are omitted due to space limitations. Here we compare the GLR tests to the MI tests on the basis of simulation for the deep hide scenario when the boundary can be accurately estimated.

\section{GLR VS. INVARIANCE PRINCIPLES}

Fig. 5 displays the magnitude of a complex valued SAR clutter image of a rural scene consisting of two clutter types (forest canopy and grass field) separated by a boundary. The deep hide target detection problem treated in this paper is to detect a target that straddles the boundary between regions $\mathrm{A}$ and $\mathrm{B}$. We make the assumptions that the complex clutter image is circular Gaussian with zero mean and that two spatial samples taken respectively from region $\mathrm{A}$ and region $B$ are uncorrelated. By centering a 1-pixel wide vertical window with fixed vertical extent at the boundary (or its estimate) in Fig. 5 and sliding it over the image from left to right we obtain a reduced image (Fig. 7) with a horizontal boundary. Any of the vectors repacked from the clutteralone image chips shown in Fig. 7 will be multivariate complex Gaussian with zero mean and covariance matrix $\mathbf{R}$ having block diagonal structure. Then by concatenating these 
vectors we obtain the measurement $\mathrm{X}=\left[\underline{x}_{1}, \ldots, \underline{x}_{n}\right]$;

$$
\mathbf{X}=a \underline{s} \underline{e}_{1}^{T}+\mathbf{N}
$$

where $\underline{s}$ is the $n$-dimensional target signature with unknown amplitude $a, \underline{e}_{1}$ is the unit vector $[1,0, \ldots, 0]^{T}$, and $\operatorname{vec}(\mathbf{N}) \sim$ $\mathcal{C N}\left(\underline{0}, \mathbf{R} \otimes \mathbf{I}_{n}\right)$, i.e. $\mathbf{N}$ follows a multivariate zero-mean complex normal distribution with covariance $\mathbf{R} \otimes \mathbf{I}_{n}$. Note that the first column $\underline{x}_{1}$ is the primary data which may contain the target. The goal is to construct a test that a given chip contains clutter alone $\left(H_{0}\right)$ vs. clutter plus target $\left(H_{1}\right)$ where target spatial structure is known. We separate the clutter scenarios into three different cases:

- Case 1: $\mathbf{R}=\left[\begin{array}{cc}\mathbf{R}_{A} & \mathbf{O} \\ \mathbf{O} & \mathbf{R}_{B}\end{array}\right]$ (totally unknown clutter in regions A and B)

- Case 2: $\mathbf{R}=\left[\begin{array}{cc}\mathbf{R}_{A} & \mathbf{O} \\ \mathbf{O} & \sigma^{2} \mathbf{I}\end{array}\right]$ (clutter known in region $\mathrm{B}$ up to variance $\sigma^{2}$ )

- Case 3: $\mathbf{R}=\left[\begin{array}{cc}\mathbf{R}_{A} & \mathbf{O} \\ \mathbf{O} & \mathbf{I}\end{array}\right]$ (clutter known exactly in region $\mathrm{B}$ )

where $\mathbf{R}_{A}>0, \mathbf{R}_{B}>0$, and $\sigma^{2}>0$.

Since there exists no uniformly most powerful test for these structured covariance matrices, the invariance principle is applied as well as the mainstay GLR method in an attempt to find good sub-optimal CFAR tests. It can be shown that the GLR tests have explicit forms only requiring rooting a complex quartic equation in the complex target amplitude parameter $a$. GLR test statistics are listed in Table 1 where the measurement matrix is partitioned as

$$
\mathbf{X}=\left[\begin{array}{l}
\mathbf{X}_{A} \\
\mathbf{X}_{B}
\end{array}\right]=\left[\begin{array}{ll}
\underline{x}_{A 1} & \mathbf{X}_{A 2} \\
\underline{x}_{B 1} & \mathbf{X}_{B 2}
\end{array}\right]
$$

where each column corresponds to pixel values in a different chip. The known target signature is $\underline{s}=\left[\underline{s}_{A}^{H} \underline{s}_{B}^{H}\right]^{H}$, and

$$
\begin{aligned}
& p\left(a, \mathbf{X}_{A}\right)=\left(\underline{x}_{A 1}-a \underline{s}_{A}\right)^{H}\left(\mathbf{X}_{A 2} \mathbf{X}_{A 2}^{H}\right)^{-1}\left(\underline{x}_{A 1}-a \underline{s}_{A}\right) \\
& q\left(a, \mathbf{X}_{B}\right)=\operatorname{tr}\left\{\left(\mathbf{X}_{B}-a \underline{s}_{B} \underline{e}_{1}^{T}\right)^{H}\left(\mathbf{X}_{B}-a \underline{s}_{B} \underline{e}_{1}^{T}\right)\right\}
\end{aligned}
$$

Here the subscripts denote the two different regions $A$ and B, $\underline{x}_{A 1}\left(m_{A} \times 1\right)$ and $\underline{x}_{B 1}\left(m_{B} \times 1\right)$ denote pixels in the chip which is being tested for containing the target.

As described in [4], the MI test is constructed by applying the likelihood ratio test to a statistic called the maximal invariant. The maximal invariant is the lowest dimension statistic summarizing the data yet preserving target vs. clutter discrimination capability for the specific uncertainty structures of cases 1,2, or 3 . Using the maximal invariant approach, Bose and Steinhardt [2] derived an MI test for Case 2, and the Kelly's test [1] can be easily modified to fit

\begin{tabular}{|c|c|c|c|}
\hline $\mathbf{R}_{A}$ & $\mathbf{R}_{B}$ & Log GLR: $\frac{1}{n} \ln \Lambda=\max _{a}\{\cdot\}$ \\
\hline \hline$?$ & $?$ & $\ln \left[\frac{1+p\left(0, \mathbf{X}_{A}\right)}{1+p\left(a, \mathbf{X}_{A}\right)}\right]+\ln \left[\frac{1+p\left(0, \mathbf{X}_{B}\right)}{1+p\left(a, \mathbf{X}_{B}\right)}\right]$ \\
\hline$?$ & $\sigma^{2} \mathbf{I}$ & $\ln \left[\frac{1+p\left(\mathbf{0}, \mathbf{X}_{A}\right)}{1+p\left(a, \mathbf{X}_{A}\right)}\right]+m_{B} \cdot \ln \left[\frac{q\left(0, \mathbf{X}_{B}\right)}{q\left(a, \mathbf{X}_{B}\right)}\right]$ \\
\hline$?$ & $\mathbf{I}$ & $\ln \left[\frac{1+p\left(\mathbf{0}, \mathbf{X}_{A}\right)}{1+p\left(a, \mathbf{X}_{A}\right)}\right]+\frac{1}{n}\left[q\left(\mathbf{0}, \mathbf{X}_{B}\right)-q\left(a, \mathbf{X}_{B}\right)\right]$ \\
\hline
\end{tabular}

Table 1. GLR tests for Case 1,2 and 3. (The notation '?' denotes 'unknown' quantity in the model)

Case 1. However, using a different function of the maximal invariant we have obtained another MI test for each case which reduces exactly to the unstructured GLR test when a

\begin{tabular}{|c|c|c|c|c|}
\hline R & $\mathbf{R}_{D}$ & {$\left[\underline{s}_{A}^{H} \underline{s}_{B}^{H}\right]$} & $\begin{array}{cc}\mathbf{K}_{A} & \mathbf{O} \\
\mathbf{O} & \mathbf{K}_{B} \\
\end{array}$ & $\begin{array}{l}\underline{x}_{A 1} \\
\underline{x}_{B 1}\end{array}$ \\
\hline $\mathbf{n}_{A}$ & $\mathrm{~N}_{B}$ & {$\left[\underline{s}_{A}^{H} \underline{s}_{B}^{H}\right]$} & {$\left[\begin{array}{cc}\mathbf{K}_{A} & \mathbf{O} \\
\mathbf{O} & \mathbf{K}_{B} \\
\end{array}\right.$} & $\begin{array}{l}\underline{s}_{A} \\
\underline{s}_{B}\end{array}$ \\
\hline$?$ & $?$ & \multicolumn{3}{|c|}{$\mathbf{K}_{A}=q_{A} \mathbf{X}_{A 2} \mathbf{X}_{A 2}^{H}, \mathbf{K}_{B}=q_{B} \mathbf{X}_{B 2} \mathbf{X}_{B 2}^{H}$} \\
\hline$?$ & $\sigma^{2} \mathbf{I}$ & \multicolumn{3}{|c|}{$\mathbf{K}_{A}=q_{A} \mathbf{X}_{A 2} \mathbf{X}_{A 2}^{H}, \mathbf{K}_{B}=v_{2} \mathbf{I}$} \\
\hline$?$ & $\mathbf{I}$ & \multicolumn{3}{|c|}{$\mathbf{K}_{A}=q_{A} \mathbf{X}_{A 2} \mathbf{X}_{A 2}^{H}, \mathbf{K}_{B}=v_{3} \mathbf{I}$} \\
\hline
\end{tabular}
target is entirely contained in one of regions A or B. The MI tests are listed in Table 2 where

$$
\begin{array}{ll}
q_{A}=1+\underline{x}_{A 1}^{H}\left(\mathbf{X}_{A 2} \mathbf{X}_{A 2}^{H}\right)^{-1} \underline{x}_{A 1} \quad, \quad v_{2}=\frac{\operatorname{tr}\left\{\mathbf{X}_{B}^{H} \mathbf{X}_{B}\right\}}{m_{B}} \\
q_{B}=1+\underline{x}_{B 1}^{H}\left(\mathbf{X}_{B 2} \mathbf{X}_{B 2}^{H}\right)^{-1} \underline{x}_{B 1} \quad, \quad v_{3}=n .
\end{array}
$$

Table 2. MI tests for Case 1, 2 and 3

\section{NUMERICAL COMPARISONS}

To compare the performance of the GLR and MI tests derived under the three structured covariance assumptions, ROC curves are generated for each case. In Figs. 1-3, the probability of detection $\left(P_{D}\right)$ is calculated empirically as a function of the probability of false alarm $\left(P_{F A}\right)$ by varying thresholds on the GLR and MI test statistics. Note that Kelly's structured test is matched to Case 1, and Bose and Steinhardt's MI test is matched to Case 2. In all cases, these figures confirm that the tests derived under the matched assumption outperform those which are mismatched.

Of particular interest, however, are the crossings in the low $P_{F A}$ regions between the GLR tests and the MI tests. In Fig. 1, we can observe the gains in $P_{D}$ of MI test 1 over GLRT 1 for $P_{F A}<0.1$. Moreover, it should be noted that the structured Kelly's test is outperformed by MI test 1 in low $P_{F A}$ and by GLRT 1 in high $P_{F A}$. Also in Case 2 (Fig. 2), both MI test 2 and GLRT 2 outperform Bose and Steinhardt's matched test and it appears that MI test 2 slightly outperforms GLRT 2 for low $P_{F A}$. These observations also hold for mismatched cases: between MI test 1 
and GLRT 1 in Case 2/3 (Figs. 2/3), and between MI test 2 and GLRT 2 in Case 1 (Figs. 1). For Case 3 (Fig. 3), the ROC curves for GLRT 2 and MI test 2 approach those of the matched GLRT 3 and MI test 3 since large number of pixels $\left(m_{B} n=60 \times 61\right)$ provide sufficiently accurate estimates of the variance in region $B$.

The relative advantage of MI vs. GLR tests are more closely investigated in Fig. 4 where we only considered Case 1, but similar results were observed for the other cases. In (a), we increased $n$ with fixed SNR and could remove the crossing. In (b), however, by increasing SNR we could only move the point of the crossing towards low $P_{F A}$. In addition, as illustrated in (c), with a large number of chips $(n)$ we could not separate MI and GLR tests by decreasing SNR.

Next, we considered an application to the real image in Fig. 5. The boundary was hand extracted and the $9 \times 7$ SLICY target from Fig. 6 was inserted so that it straddles the boundary. With the realigned image in Fig. 7, MI test 1 and GLRT 1 statistics are calculated and maximized pixel by pixel (Fig. 8). Both tests detected the target in column 300 , yet in this experiment the GLRT 1 statistic exhibits slightly higher detectiblity at the true target location.

\section{CONCLUSION}

The deep hide scenario considered in this paper complicates the design of optimal target detectors. This scenario gives rise to block diagonal constraints imposed by the clutter covariance structure. Both GLR and MI tests can be derived under these constraints. Numerical results indicate that neither GLR nor MI tests dominate the other in terms of ROC performance. The GLRT outperforms the MI test only when high estimator accuracy is attainable, e.g. for a large number of samples, but otherwise MI test is better especially in low $P_{F A}$. Therefore, MI test not only plays an important role as an alternative to GLRT, but also has the desirable property of reliable performance in low $P_{F A}$ with a small number of data.

\section{REFERENCES}

[1] E. J. Kelly, "An adaptive detection algorithm," IEEE Trans. Aerosp. Electron. Syst., vol. AES-22, pp. 115-127, Mar. 1986.

[2] S. Bose and A. O. Steinhardt, "A maximal invariant framework for adaptive detection with structured and unstructured covariance matrices," IEEE Trans. Sig. Proc., vol. 43, no. 9, pp. 2164-2175, Sep. 1995

[3] A. O. Hero and C. Guillouet, "Robust detection of SAR/IR targets via invariance," 1997 ICIP, Santa Barbara, CA, vol. 3, pp. 472-475, Oct. 1997.

[4] T. Kariya and B. K. Sinha, Robustness of Statistical Tests, Academic Press, San Diego, 1989.

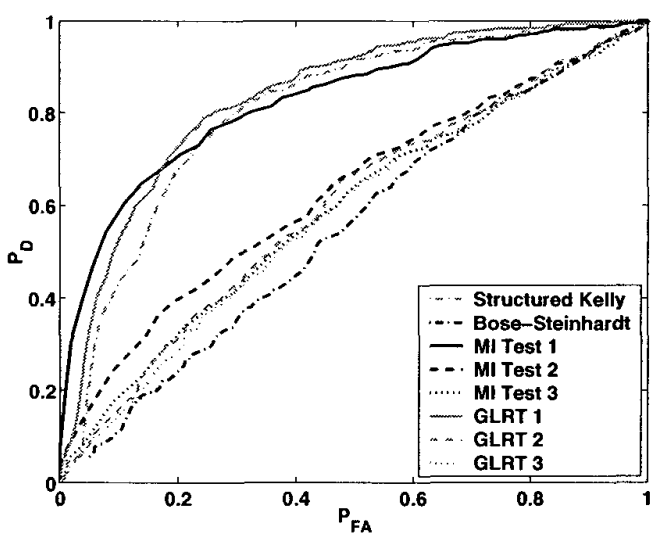

Fig. 1. ROC curves for Case 1 with $S N R=22 d B\left(m_{A}=\right.$ $50, m_{B}=50, n=51$ ).

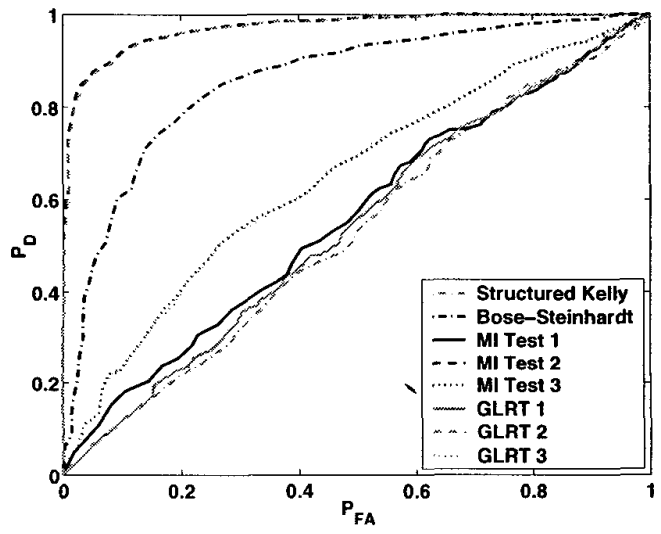

Fig. 2. ROC curves for Case 2 with $S N R=10 d B\left(m_{A}=\right.$ $40, m_{B}=60, n=61$ ).

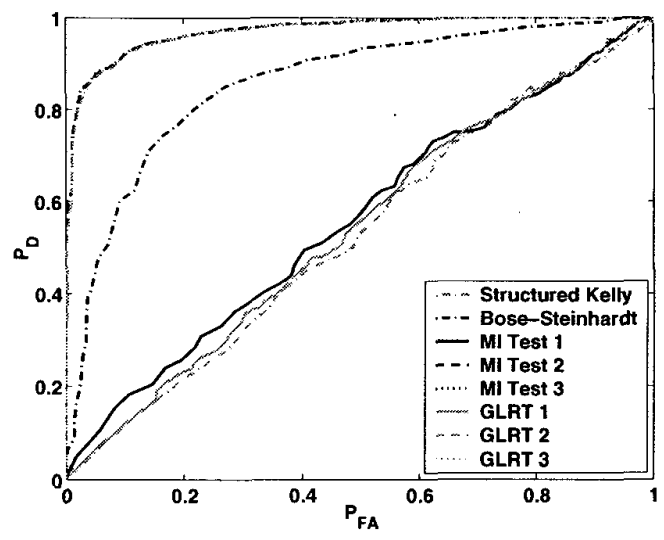

Fig. 3. ROC curves for Case 3 with $S N R=10 d B\left(m_{A}=\right.$ $\left.40, m_{B}=60, n=61\right)$. 


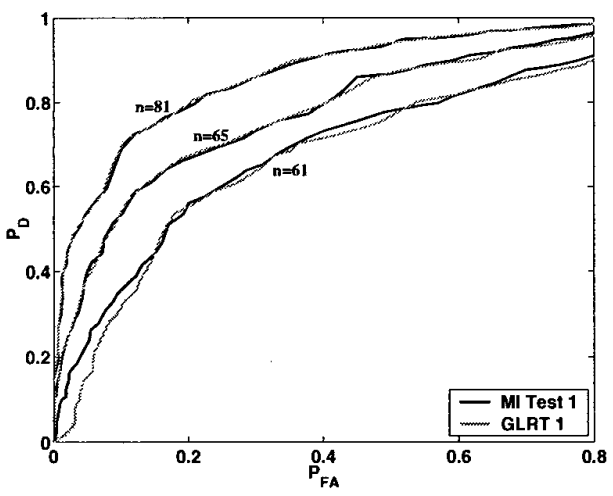

(a) $S N R=7 d B$

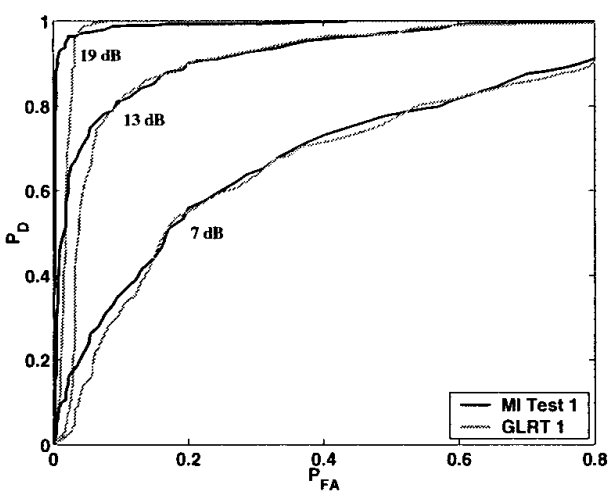

(b) $n=61$

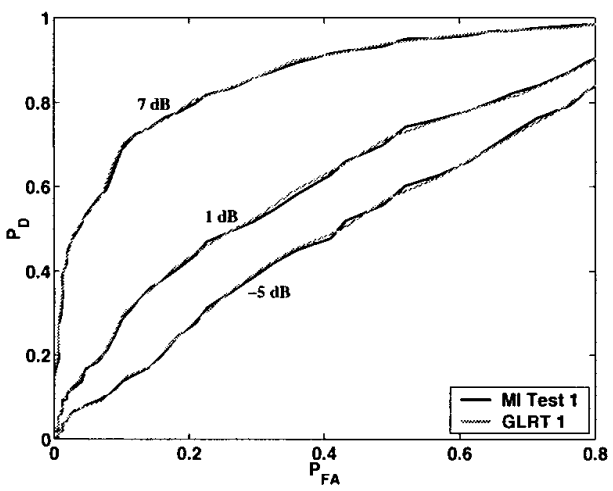

(c) $n=81$

Fig. 4. Comparison of GLR and MI tests for Case 1 by (a) varying $n$ with fixed SNR, (b) increasing $S N R$ with small $n$, and (c) decreasing SNR with large $n\left(m_{A}=60, m_{B}=40\right)$.

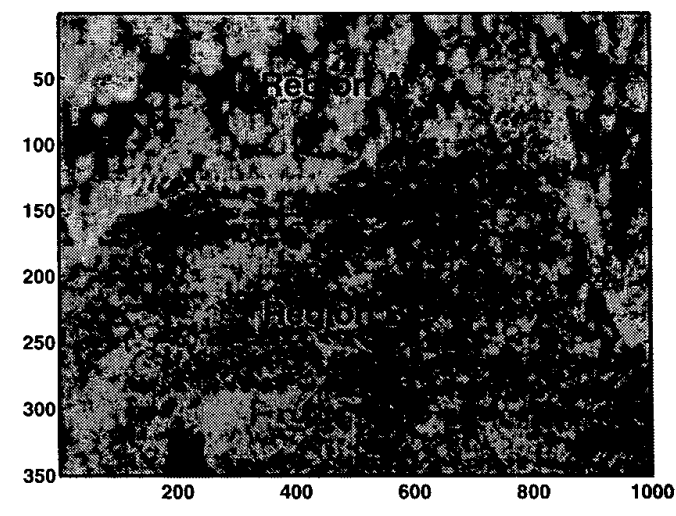

Fig. 5. SAR clutter image with SLICY target in the boundary at column 300 . The data from which this rural scene is reproduced is downloaded from the MSTAR SAR database at the Center for Imaging Science (www.cis.jhu.edu).

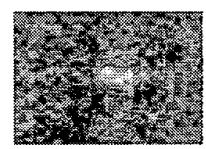

Fig. 6. SLICY canonical target image at azimuth $=163^{\circ}$ and elevation $=39^{\circ}$ obtained from the MSTAR database.

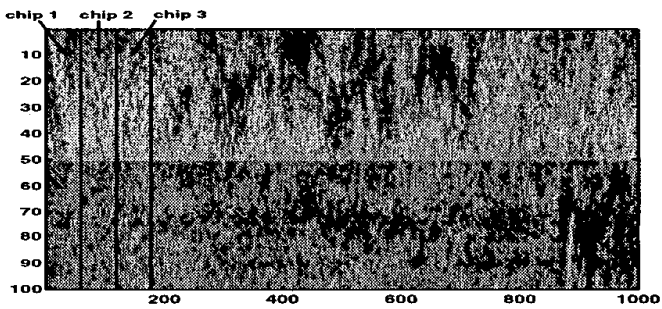

Fig. 7. Image realigned along the extracted boundary. SLICY target is located at column 300 .

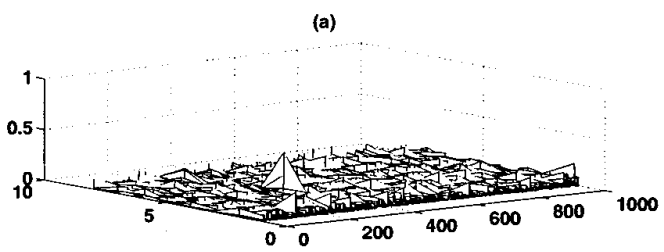

(b)

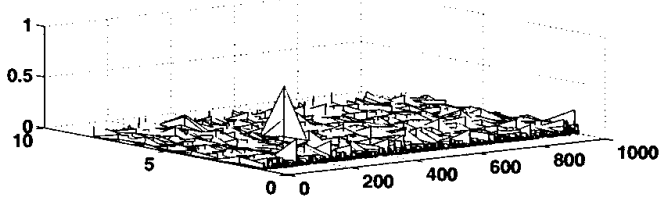

Fig. 8. Test values obtained along the boundary by (a) MI test 1 and (b) GLRT 1. 